\title{
Prevalence of Toxocara Infection in Schoolchildren from the Butantã Region, São Paulo, Brazil
}

\author{
Jussara MS Alderete, Cristina MA Jacob/ ${ }^{+}$, Antonio C Pastorino, Guita R Elefant*, \\ Ana PM Castro, Angela BF Fomin, Pedro P Chieffi**
}

\begin{abstract}
Unidade de Alergia e Imunologia, Departamento de Pediatria, Faculdade de Medicina, Universidade de São Paulo, Av. Dr. Enéas Carvalho Aguiar 647, 05403-900 São Paulo, SP, Brasil *Laboratório de Soroepidemiologia e Imunobiologia Celular e Molecular

**Laboratório de Imunopatologia da Esquistossomose, Instituto de Medicina Tropical de São Paulo, São Paulo, SP, Brasil
\end{abstract}

Visceral larva migrans syndrome by Toxocara affects mainly children between 2 and 5 years of age, it is generally asymptomatic, and the seroprevalence varies from 3 to $86 \%$ in different countries. A total of 399 schoolchildren from 14 public schools of the Butantã region, São Paulo city, Brazil, were evaluated by Toxocara serology (enzymelinked immunosorbent assay). Epidemiological data to the Toxocara infection obtained from a protocol were submitted to multiple logistic regression analysis for a risk profile definition. Blood was collected on filter paper by finger puncture, with all samples tested in duplicate. Considering titers $\geq 1 / 160$ as positive, the seroprevalence obtained was $38.8 \%$. Among infected children, the mean age was 9.4 years, with a similar distribution between genders. A significant association was observed with the presence of onychophagia, residence with a dirty backyard, living in a slum, previous wheezing episodes, school attended, and family income $(p<0.05)$. All data, except "living in a slum", were considered to be determinant of a risk profile for the acquisition of Toxocara infection. A monthly income $\geq 5$ minimum salaries represented a protective factor, although of low relevance. Toxocara eggs were found in at least one of the soil samples obtained from five schools, with high prevalence of Toxocara infections, indicating the frequent soil contamination by this agent.

Key words: Toxocara - seroprevalence - schoolchildren - São Paulo - Brazil

Visceral larva migrans is a clinical syndrome caused by the migration and persistence of nematode larvae in the tissues of non-habitual hosts (Glickman \& Shofer 1987), with species of the genus Toxocara being most frequently involved (Beaver 1956). The disease affects mainly children under 5 years of age, and clinical manifestations include visceral and ocular toxocariasis. Covert toxocariasis and atypical forms have also been described. Visceral toxocariasis is the most common form of clinical presentation and is characterized by fever, hepatomegaly, pulmonary manifestations, chronic eosinophilia, leukocytosis, hypergammaglobulinemia, and high isohemagglutinin titers (Schantz 1989).

The method of choice for the toxocariasis diagnosis is excretion-secretion antigen enzyme-linked immunosorbent assay (ELISA) (Schantz \& Glickman 1983), which shows $78.3 \%$ of sensitivity and $92.3 \%$ of specificity (Glickman et al. 1978).

The prevalence of toxocariasis is higher in tropical and developing countries and is generally associated with low socioeconomic level (Thompson et al. 1986, Lynch et al. 1988), with rates ranging from 1\% in Spain (Portus et al. 1989 ) to $86 \%$ in Santa Lucia (Thompson et al. 1986). In Brazil, Chieffi et al (1990), evaluating 2025 sera obtained

Research supported by Fapesp, Nr 3667- 4/1998/03667- 4. ${ }^{+}$Corresponding author. Fax: +55-11-3064.6483. E-mail: cristmaj@icr.honet.usp.br

Received 9 February 2002

Accepted 30 April 2003 from individuals of different age groups found $3.6 \%$ of positive samples, and Caseiro (1996), studying 2056 schoolchildren, aged 4 to 15 years, observed $24.7 \%$ of positive samples.

In the present study, we determined the seroprevalence of Toxocara sp. infection and its association with epidemiological data in a population of schoolchildren from the Butantã region, São Paulo city, to identify probable risk factors for Toxocara infection in this population.

\section{MATERIALS AND METHODS}

The study, carried out from May 1998 to October 1999, included children enrolled from the 1 st to the 4 th grade of 15 public schools, numbered 1 to 14 . The school number 8 was excluded because was characterized as an orphanage, a fact that could compromise the reliability of epidemiological data.

From a total of 7755 schoolchildren on the class list provided, were selected 494 children through random criteria, using a sampling interval. The sample size was based on an expected prevalence of $15 \%$ with a precision of $6 \%$ and an alpha value of $5 \%$.

The inclusion criteria were: free informed consent signed by the parents or responsible person; a properly filled out questionnaire about epidemiological data answered by the parents; absence of interference by school staff in the children selection; and adequate identification of the child on both the field spreadsheet and the questionnaire answered by the parents.

After the application of all criteria, composed of the sample was 399 children and adolescents, including 196 boys (49.1\%) and 203 girls (50.9\%) with a male:female ra- 
tio of 0.96 . The mean age was 9.4 years (range: 7 to 16 years), with $63.9 \%$ of the population aged less than 10 years. The schoolchildren distribution according to school varies from 3.5 to $10.8 \%$.

At the beginning of the study, all children were submitted to an epidemiological and clinical protocol and Toxocara serology (ELISA), using disposable lancets $\left(\right.$ Feather ${ }^{\circledR}$ ) and blood collection on filter paper (Whatman ${ }^{\circledR}$ $\mathrm{Nr} 3$ ). These papers were booklets in groups of $10,1 \mathrm{~cm}$ long strips separated by cellophane paper, and field spreadsheets individualized for each school.

Blood was collected at the school from the digital pulp of the third finger of the hand opposite to that used for writing, after $70 \%$ alcohol usage. The first drop was discarded with dry cotton and the strip was then slid over the finger of the child, visualizing the uniform stain formed by the blood absorbed on the filter paper. The booklets were dried at room temperature and transported on the same day to the Laboratorial Section of Seroepidemiology and Cellular and Molecular Immunology, Institute of Tropical Medicine, Faculty of Medicine, University of São Paulo (LIM48 - Laboratory of Medical Investigation) where serology was done.

ELISA- Based on the technique described by De Savigny et al. (1979), modified by Bach-Rizzatti (1984).

Eluate - The filter papers containing the collected blood were cut into $1 \mathrm{~cm}^{2}$ samples, $330 \mu 1$ of $0.01 \mathrm{M}$ phosphate buffered saline (PBS), pH 7.2, was added (corresponding to a $1 / 20$ dilution), and the papers were incubated for $18 \mathrm{~h}$ at $4^{\circ} \mathrm{C}$. Absorption with Ascaris suum antigen extract at a final dilution of $1 / 160$ was then carried out for $30 \mathrm{~min}$ at $37^{\circ} \mathrm{C}$. All eluates were tested in duplicate.

Sera - Using the 1/160 dilution the sera were incubated with $\mathrm{A}$. suum antigen extract for $30 \mathrm{~min}$ at $37^{\circ} \mathrm{C}$ and then submitted to serial dilutions (ratio of 2) until 1/10 240.

Polystyrene plates were sensitized with $100 \mu 1$ Toxocara excretion-secretion antigen (TES) $(2 \mu \mathrm{g} / \mathrm{ml})$ in $0.01 \mathrm{M}$ PBS, pH 7.2. After washing with PBS-Tween, the reaction was blocked with $1 \%$ bovine albumin in PBS-Tween. The plates were washed and incubated with peroxidase-labeled human anti-IgG conjugate in PBS-Tween. After washing, $100 \mu \mathrm{l}$ of the chromogen solution containing $0.015 \%$ hydrogen peroxide was added. After incubation, the enzymatic reaction was stopped with $50 \mu \mathrm{l}$ of $2 \mathrm{M}$ sulfuric acid and the optical density was determined at $492 \mathrm{~nm}$. Sera and/or eluates presenting an optical density of less than 0.5 were considered to be non-reactive.

The criterion adopted for the diagnosis of Toxocara infection was a serology (ELISA) titer $\geq 1 / 160$ (Chieffi et al. 1990).

Determination of Toxocara eggs in soil samples obtained from the schools and their surroundings - After the high prevalence had been known, we decided to collect soil material at 5 schools, being 3 with higher seroprevalence and 2 with lower prevalence. The soil samples were collected at about eight different points at a depth of 10-15 cm and an inclination of about $45^{\circ}$ and sent to the Institute of Tropical Medicine for analysis. About 15 to $20 \mathrm{~g}$ of each soil sample was transferred to sedimentation chalices, homogenized, and $20 \mathrm{ml}$ of a $0.1 \mathrm{~N}$
$\mathrm{KOH}$ solution was added. After $20 \mathrm{~min}, 200 \mathrm{ml}$ of hot, hyper saturated $\mathrm{ZnSO}_{4}$ was added in order to obtain a fluid with a density of 1.3. Aliquots of the surface film of the fluid were collected, transferred to microscopic slides, covered with $24 \times 24 \mathrm{~mm}$ cover slips, and examined under a light microscope at $100 \mathrm{X}$ magnification.

Statistical analysis - Initially, the independent variables were evaluated according to seropositivity through univariated analysis, using conventional chi-square test (Rosner 1986).

The variables showing statistical significance $(\mathrm{p}<$ 0.05 ) upon univariate analysis were used to fit a multiple logistic regression model with stepwise selection of the variables (Hosmer \& Lemeshow 1989), in order to identify the factors determining a risk profile for Toxocara infection in this population. For this analysis, the schools were divided into two groups according to schools with a seroprevalence $>40 \%$ (group 1 ) and $\leq 40 \%$ (group 2 ).

For analysis, the group with variable income was defined as not having a fixed salary, with the income coming from informal work. For multiple regression analysis, the family income was divided into two categories: family income $<5$ and $\geq 5$ minimum salaries.

A p value $\leq 0.05$ was considered to be significant. The Epi Info 6.04 and SPSS programs were used for analysis of the data.

The study was approved by the Ethics and Research Committee of Pediatric Department, and by the Ethics Committee for the Analysis of Research Projects of the Clinical Hospital, Faculty of Medicine, University of São Paulo.

\section{RESULTS}

With respect to family income, $39.4 \%$ of the families had monthly income $\leq 3$ minimum salaries. Wheezing reference was present in $33.6 \%$ of the schoolchildren's population, with this finding occurring during the last 6 months in $27.3 \%$. Only $3 \%$ of the population reported habits of geophagy, while onychophagy was reported by $44.9 \%$ of the schoolchildren.

When asked about a suspicion of other parasitic infection in their child, $64.9 \%$ of the parents or responsible persons answered affirmatively, $22.6 \%$ reported that their children were undergoing microscopic examination of the stools, and $32.3 \%$ reported parasite treatment during the last 6 months.

Almost half the population had a dog at home (44.5\%) and $14.2 \%$ of them reported contact with a pet. Contact with cats was reported by $16.3 \%$ of the population.

Of the 399 children, $34.8 \%$ lived in a slum and an earth backyard was present in $30.8 \%$ of the homes.

Toxocara serology (ELISA) was positive in $38.8 \%$ of the schoolchildren $(\mathrm{n}=155)$. The distribution of the titers is shown in Table I. No statistically significant difference in age, sex or school grade was observed between the seropositive and seronegative populations.

Analysis of seroprevalence according to school (Fig. 1) showed that this variable may have a discriminating character $(p=0.005)$. At schools 2, 4, 9, 11, 13 and 15, more than $40 \%$ of the schoolchildren presented positive serology. 
TABLE I

Distribution of Toxocara serologic titers (ELISA) in 399 schoolchildren, Butantã region, São Paulo

\begin{tabular}{lcc}
\hline Serologic titers & Nr of children & $\%$ \\
\hline Negative & 244 & 61.2 \\
$1 / 160$ & 54 & 13.5 \\
$1 / 320$ & 45 & 11.3 \\
$1 / 640$ & 34 & 8.5 \\
$1 / 1280$ & 22 & 5.5 \\
\hline Total & 399 & 100 \\
\hline
\end{tabular}

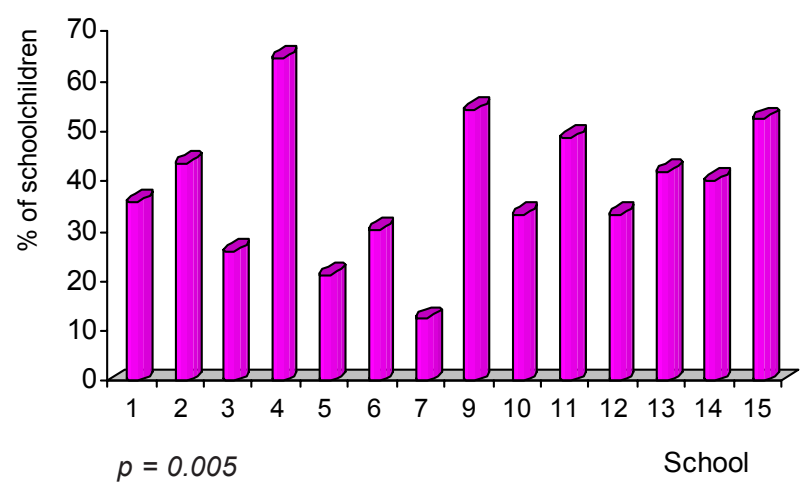

Fig. 1: distribution of 394 schoolchildren with Toxocara-positive serology (ELISA) from the Butantã region, São Paulo, according to school attended.

At the school with the highest seroprevalence (number 4 ), $70.9 \%$ of the children lived in a slum and the parents or responsible persons suspected of other parasitic infection in $87.1 \%$ of the children.

Schools 4, 9, 11 and 15, which presented the highest seropositivity, also showed higher percentages of children living in a house with a dirt backyard, and children with a reference to wheezing, presence of onychophagy, and suspicion of other parasitic infection. All schools with seroprevalence rates $>40 \%(2,4,9,11,13$ and 15) were located in a district characterized by poor socioeconomic level. Children from the school 4 living in a large regional slum, a fact that may have contributed to the highest seroprevalence data differing from those of the other schools in the district. The statistically significant associations are shown in Table II.

The presence of infection was inversely proportional to the monthly family income of the infected children (Fig. 2 ), with this relationship being statistically significant ( $p$ $=0.03$ ). Analysis of the figure revealed that the frequency of Toxocara infection decreased with increasing of monthly family income.

In order to identify possible factors that may predispose to Toxocara infection, multiple regression analysis was applied to those variables that were significant upon comparison between the infected and non-infected population. Analysis revealed the following factors as being important (Table III): school attended by the child, reference of wheezing, presence of onychophagy and the presence of a dirty backyard at the children's residence. These factors have represented a risk profile for the acquisition of Toxocara infection among this population. Family income had not been included, because this date could be unreliable, and it was analyzed separately.

Regarding to the determination of Toxocara eggs in soil samples obtained from five different schools, the eggs had been found at least in one of the samples collected from all schools. Viable eggs were found only at school number 9. In two other schools, larvae of Ancylostoma caninum and Ascaris lumbricoides eggs had also been found.

\section{TABLE II}

Relationship between Toxocara serology and epidemiological data reported by schoolchildren from the Butantã region, São Paulo

\begin{tabular}{|c|c|c|c|c|}
\hline \multirow[b]{2}{*}{ Epidemiological } & \multirow[b]{2}{*}{ Data } & \multicolumn{2}{|c|}{ Serology \% } & \multirow[b]{2}{*}{$\mathrm{P}$} \\
\hline & & Positive & Negative & \\
\hline \multirow{2}{*}{$\begin{array}{l}\text { Reference to wheezing } \\
(\mathrm{n}=391)\end{array}$} & Yes & 50.7 & 49.2 & \multirow[t]{2}{*}{0.0004} \\
\hline & No & 32.3 & 67.7 & \\
\hline \multirow[t]{2}{*}{ Onychophagy $(n=394)$} & Yes & 49.2 & 50.8 & \multirow[t]{2}{*}{0.0001} \\
\hline & No & 29.8 & 70.2 & \\
\hline \multirow{2}{*}{$\begin{array}{l}\text { Family suspicion of } \\
\text { verminosis }(n=387)\end{array}$} & Yes & 44.4 & 55.6 & \multirow[t]{2}{*}{0.0012} \\
\hline & No & 27.3 & 72.7 & \\
\hline \multirow{2}{*}{$\begin{array}{l}\text { Living in a shanty town } \\
(\mathrm{n}=394)\end{array}$} & Yes & 46 & 54 & \multirow[t]{2}{*}{0.0003} \\
\hline & No & 34.5 & 65.5 & \\
\hline \multirow{2}{*}{$\begin{array}{l}\text { Residence with a } \\
\text { dirt backyart }(\mathrm{n}=395)\end{array}$} & Yes & 49.6 & 50.4 & \multirow[t]{2}{*}{0.0037} \\
\hline & No & 34.2 & 65.8 & \\
\hline
\end{tabular}

TABLE III

Risk profile for Toxocara infection in 328 schoolchildren, Butantã region, São Paulo, in 1998

\begin{tabular}{lccc}
\hline Variable & $\begin{array}{c}\text { Odds } \\
\text { ratio }\end{array}$ & $\begin{array}{c}\text { Confidence } \\
\text { interval }\end{array}$ & $\mathrm{p}$ \\
\hline Attending a group 1 school & 2,492 & $1,516-4,098$ & 0.0003 \\
Reference to wheezing & 2,299 & $1,389-3,805$ & 0.0012 \\
Presence of onychophagy & 2,764 & $1,688-4,527$ & 0.0001 \\
Presence with a dirt backyard & 1,715 & $1,011-2,910$ & 0.0454 \\
$\begin{array}{l}\text { Family income } \geq 5 \text { minimun } \\
\text { salaries }\end{array}$ & 0,853 & $0,738-0986$ & 0.0319 \\
& & & \\
\end{tabular}

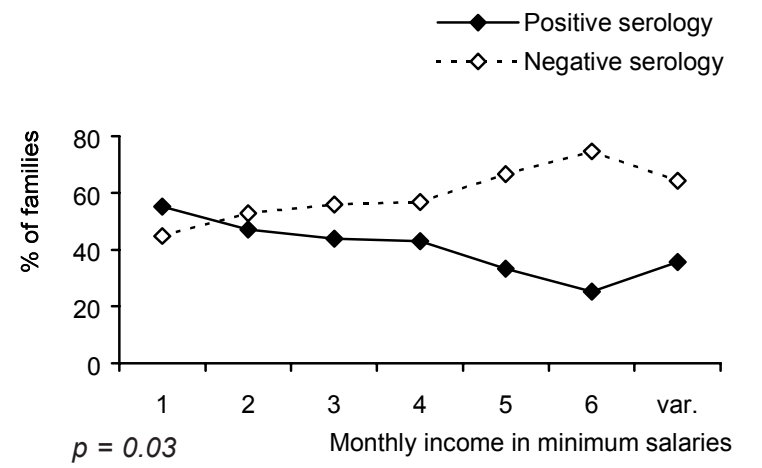

Fig. 2: Toxocara serology according to monthly income in 380 schoolchildren from the Butantã region, São Paulo, in 1998. 


\section{DISCUSSION}

Human toxocariasis is still a poorly diagnosed disease, especially in places with conditions, which favors its development, and it is largely unknown either to health professionals or the general population.

In the present study, the mean age of seropositive children was slightly higher than that reported in other studies in the literature, but it is necessary to point out that the evaluated population is attending from the first primary grade up to the fourth. Caseiro (1996) observed $27.6 \%$ of prevalence rates ranging from 7 to 9 years old and $18.9 \%$ ranging 10 to 12 years old. Bundy et al. (1987) suggested that the prevalence rates of infection may provide some indication of an age/infection relationship and that the rates found for the age group under 5 years are probably a true reflection of the infection acquisition rate, since this population had not been infected at birth.

No statistically significant difference regarding sex was observed at the present study. Literature data have reported a predominance of $T$. canis infection in males, which may be explained by differences in the playing and social behaviors of boys, resulting in an increased exposure to Toxocara eggs (Overgaauw 1997).

A significant association was observed between onychophagy and positive serology. Onychophagy may be an epidemiological characteristic of schoolchildren corresponding to geophagy in younger children, and it should therefore be considered.

The statistically significant association observed between low family incomes and seropositivity is in agreement with various serologic studies showing a strong association between Toxocara antibody titers and socioeconomic indicators such as level of schooling and head family income (Glickman \& Schantz 1981, Herrmann et al. 1985). Precarious sanitarian and hygienic conditions, and probably other factors associated to low income, facilitate the transmission of Toxocara infection as well as of many other diseases.

In the present study, a significant difference in the seroprevalence was observed among the different schools analyzed. One noteworthy aspect is the fact that the schools with the highest seropositivity rates, except for school 4, were located in the poorest socioeconomic conditions district. However, at school 4 many children came from a large neighboring slum, with probable consequent maintenance of the factors favoring the development of toxocariasis. Caseiro (1996), in another municipality in the same state, also found a higher prevalence in regions with low acquisitive power, a low urbanization index and part of the population without access to sanitarian conditions, with positivity ranging from 27.7 to $35.1 \%$.

In the present study, the highest seropositive school (school 4) also showed the highest rate of children living in houses with a dirty backyard (non-significant correlation), a highest percentage of parents suspecting others parasitic infection in their children, and the largest number of children living in a slum.

In relation to the factors that lead to a situation of higher risk for the acquisition of Toxocara infection, as no cause-effect relationship was established, we have chosen to name them as a profile risk for the acquisition of infection in this population, which may have a value as separately as in association. Monthly family income probably generates some social, cultural and environmental conditions which are more favorable to Toxocara infection, but this information is not trustworthy. A dirty backyard is probably more common in low-income families houses and the children of these families probably attend schools located in inadequate areas. Therefore, based on the present results obtained by multiple regression analysis, the profile of a child with a higher chance of being infected with Toxocara can be defined by the following aspects: the ones attending group 1 , with a dirty backyard at home, presenting the habit of onychophagy, and referring wheezing.

Regarding risk profile, the schools here analyzed probably represent more an aggregation factor of children who share a similar life-condition than a dangerous factor to Toxocara infection.

Although Toxocara eggs have been found in the soil of some schools, it could not be confirmed that these children had been infected at the schools. It would be important to extend the investigation to their homes or playing places parks. Most probably, as it has been demonstrated in several studies, the rate of environmental contamination with Toxocara eggs is high, in addition to the presence of a large number of strayed dogs in these peripheral neighborhoods. The school probably represents a meeting place for individuals sharing similar environmental life conditions determined by their socioeconomic level. It is also important to note that the methods used to recover T. canis eggs are of low sensitivity (Dada \& Lindquist 1979, Collins \& Moore 1982), indicating the frequent underestimation of the environmental contamination.

In the present study, we have observed a high seroprevalence $(38.8 \%)$ compared to literature data. In fact, although Toxocara serology (ELISA) is a better and an important method for the toxocariasis diagnosis, the meaning of the antibody titers remains unknown. Many studies showed that antibodies persist for long periods of time due to repeated antigen stimulation resulting from the persistence of live larvae in tissues (Beaver 1969) or from periodic reinfection (Glickman \& Schantz 1981).

The finding of asymptomatic children with positive serology, even at high titers, suggests that the infection is not always related to clinical manifestations. Schantz (1989) pointed out that the clinician, when interpreting the serologic findings of the patient, should be aware of the fact that a measurable titer does not prove a causal relationship between $T$. canis infection and clinical symptoms at that time. In many populations, the occurrence of a low, but variable, positive ELISA titer apparently reflects the prevalence of asymptomatic toxocariasis.

The interpretation of seroprevalence data still remains very difficult due to the use of different cut-off titers by various researchers and the difficulty in evaluating the relationship between titer level, infection and clinical findings of the disease. Prevalence data based on hospitalized patient's serum, blood donors or high-risk groups 
tend to introduce bias and do not provide accurate population data (Holland et al. 1995).

The population studied here was from an urban area with a high demographic density, a fact favoring the existence of a significant number of individuals with anti-Toxocara antibodies as well as a closer man-dog contact and high exposure to dog feces-contaminated environments (Chieffi et al. 1990).

Although the population evaluated in this study does not represent a high risk age group, the prevalence observed led us to conclude that there is probably a large environmental contamination with Toxocara eggs. Further studies are required for a better evaluation of soil contamination and the consequence of this disease in the population.

\section{ACKNOWLEDGMENT}

To Professor Cláudio Leone for his suggestions.

\section{REFERENCES}

Bach-Rizzatti BC 1984. Desenvolvimento de Teste Imunoenzimático, ELISA, para o Diagnóstico da Toxocaríase Humana, MSc Thesis, Faculdade de Ciências Farmacêuticas, Universidade de São Paulo, São Paulo, 105 pp.

Beaver PC 1956. Parasitological Reviews - Larva Migrans. Exp Parasitol 5: 587-621.

Beaver PC 1969. The nature of visceral larva migrans. J Parasitol 55: 3-12.

Bundy DAP, Thompson DE, Robertson BD, Cooper ES 1987. Age-relationships of Toxocara canis seropositivity and geohelminth infection prevalence in two communities in St. Lucia, West Indies. Trop Med Parasitol 38: 309-312.

Caseiro MM 1996. Sindrome de Larva Migrans Visceral Causada por Larvas de Toxocara canis, no Município de Santos, MSc Thesis, Faculdade de Medicina, Universidade de São Paulo, São Paulo, 121 pp.

Chieffi PP, Ueda M, Camargo ED, de Souza AM, Guedes ML, Gerbi LJ, Spir M, Moreira AS 1990. Visceral larva migrans: a seroepidemiological survey in five municipalities of São Paulo state, Brazil. Rev Inst Med Trop São Paulo 32: 204210.

Collins GH, Moore J 1982. Soil survey for eggs of Toxocara species. Ann Trop Med Parasitol 76: 579-580.

Dada BJO, Lindquist WD 1979. Studies on flotation techniques for the recovery of helminths eggs from soil and the prevalence of eggs of Toxocara spp. in some Kansas public places. J Am Vet Med Assoc 174: 1208-1210.

De Savigny DH, Voller A, Woodruff AW 1979. Toxocariasis: serological diagnosis by enzyme immunoassay. J Clin Pathol 32: 284-288.

Glickman LT, Schantz PM 1981. Epidemiology and pathogenesis of zoonotic toxocariasis. Epidemiol Rev 3: 230-250.

Glickman LT, Shofer FS 1987. Zoonotic visceral and ocular larva migrans. Vet Clin North Am 17: 39-53.

Glickman LT, Schantz PM, Dombroske R, Cypess R 1978. Evaluation of serodiagnostic tests for visceral larva migrans. Am J Trop Med Hyg 27: 492-498.

Hermann N, Glickman LT, Schantz PM 1985. Seroprevalence of zoonotic toxocariasis in the United States: 1971-1973. Am J Epidemiol 122: 890-896.

Holland CV, O’Lorcain P, Taylor MR, Kelly A 1995. Seroepidemiology of toxocariasis in school children. Parasitology 110: 535-545.

Hosmer DW, Lemeshow S 1989. Applied Logistic Regression, John Wiley \& Sons, New York, 307 pp.

Lynch NR, Wilkes LK, Hodgen AN, Turner KJ 1988. Specifity of Toxocara ELISA in tropical populations. Parasite Immunol 10: 323-327.

Overgaauw PA 1997. Aspects of Toxocara epidemiology: toxocarosis in dogs and cats. Crit Rev Microbiol 23: 233251.

Portus M, Riera C, Prats G 1989. A serological survey of toxocariasis in patients and healthy donors in Barcelona (Spain). Eur J Epidemiol 5: 224-227.

Rosner B 1990. Fundamentals of Biostatistics, 3rd ed., PWS Publishers, Boston, 655 pp.

Schantz PM 1989. Toxocara larva migrans now. Am J Trop Med Hyg 41: 9-122.

Schantz PM, Glickman LT 1983. Ascarids of cats and dogs: a public health and veterinary medicine problem. Bol Oficina Sanit Panam 94: 571-586.

Thompson DE, Bundy DAP, Cooper ES, Schantz PM 1986. Epidemiological characteristics of Toxocara canis zoonotic infection of children in a Caribbean community. Bull WHO 64: 283-290. 